\title{
Implementation of a Behavioral Economics Electronic Health Record (BE-EHR) Module to Reduce Overtreatment of Diabetes in Older Adults
}

\author{
Hayley M. Belli, $P h D^{7}$ (D), Sara K. Chokshi, $P h D^{2}$, Roshini Hegde, $M S^{3}$, \\ Andrea B. Troxel, ScD' , Saul Blecker, MD ${ }^{2,4}$, Paul A. Testa, MD ${ }^{5,6}$, Judd Anderman, MS 5 , \\ Christina Wong, $B S^{5}$, and Devin M. Mann, $M D^{2,4,5}$
}

\begin{abstract}
'Division of Biostatistics, Department of Population Health, New York University School of Medicine, New York, NY, USA; ${ }^{2}$ Division of Healthcare Delivery Science, Department of Population Health, New York University School of Medicine, New York, NY, USA; ${ }^{3}$ Fern Health, New York, NY, USA; ${ }^{4}$ Department of Medicine, New York University School of Medicine, New York, NY, USA; ${ }^{5}$ Medical Center Information Technology, NYU Langone Health, New York, NY, USA; 'Department of Emergency Medicine, New York University School of Medicine, New York, NY, USA.
\end{abstract}

\begin{abstract}
BACKGROUND: Intensive glycemic control is of unclear benefit and carries increased risk for older adults with diabetes. The American Geriatrics Society's (AGS) Choosing Wisely (CW) guideline promotes less aggressive glycemic targets and reduction in pharmacologic therapy for older adults with type II diabetes. Meanwhile, behavioral economic (BE) approaches offer promise in influencing hard-to-change behavior, and previous studies have shown the benefits of using electronic health record (EHR) technology to encourage guideline adherence.

OBJECTIVE: This study aimed to develop and pilot test an intervention that leverages BE with EHR technology to promote appropriate diabetes management in older adults.
\end{abstract}

DESIGN: A pilot study within the New York University Langone Health (NYULH) EHR and Epic system to deliver BE-inspired nudges at five NYULH clinics at varying time points from July 12, 2018, through October 31, 2019.

PARTICIPANTS: Clinicians across five practices in the NYULH system whose patients were older adults (age 76 and older) with type II diabetes.

INTERVENTIONS: A BE-EHR module comprising six nudges was developed through a series of design workshops, interviews, user-testing sessions, and clinic visits. $\mathrm{BE}$ principles utilized in the nudges include framing, social norming, accountable justification, defaults, affirmation, and gamification.

MAIN MEASURES: Patient-level CW compliance.

KEY RESULTS: CW compliance increased 5.1\% from a 16-week interval at baseline to a 16-week interval post intervention. From February 14 to June 5, 2018 (prior to

Prior Presentations: This work was previously presented at the Society of General Internal Medicine 2019 Annual Meeting, Society for Behavioral Medicine 2019 Annual Meeting, and the 2018 Conference on the Science of Dissemination and Implementation (all three located in Washington, D.C.), as well as at the 2019 Biannual Meeting of the International Medical Informatics Association (MedInfo) in Lyon, France.

Electronic supplementary material The online version of this article (https://doi.org/10.1007/s11606-020-06119-z) contains supplementary material, which is available to authorized users.

Received January 18, 2020

Accepted August 6, 2020

Published online September 3, 2020 the first nudge launch in Vanguard clinics), CW compliance for 1278 patients was mean (95\% CI)-16.1\% (14.1\%, 18.1\%). From July 3 to October 22, 2019 (after BE-EHR module launch at all five clinics), CW compliance for 680 patients was $21.2 \%(18.1 \%, 24.3 \%)$.

CONCLUSIONS: The BE-EHR module shows promise for promoting the AGS CW guideline and improving diabetes management in older adults. A randomized controlled trial will commence to test the effectiveness of the intervention across 66 NYULH clinics.

NIH TRIAL REGISTRY NUMBER: NCT03409523

KEY WORDS: diabetes; behavioral economics; electronic health records; clinical decision support.

J Gen Intern Med 35(11):3254-61

DOI: $10.1007 / \mathrm{s} 11606-020-06119-z$

(C) Society of General Internal Medicine 2020

\section{INTRODUCTION}

Intensive glycemic control is of unclear benefit and carries increased risk for older adults with diabetes. A number of randomized controlled trials, including ACCORD, ${ }^{1}$ AD$\mathrm{VANCE},{ }^{2}$ and $\mathrm{VADT},{ }^{3}$ found that intensive glycemic control did not reduce risk of diabetes complications including myocardial infarction or stroke. These trials indicated that tight glycemic control is primarily beneficial to patients with newly diagnosed diabetes and a long life expectancy (LE), characteristics that do not apply to most older patients. ${ }^{4}$ Furthermore, these trials demonstrated the potential for harm with tight glycemic control, notably increased risk of hypoglycemia, ${ }^{1,2}$ including emergent hospitalization and neurologic complications, ${ }^{5-9}$ a suggestion of increased all-cause mortality, ${ }^{1}$ and potential increased risk of polypharmacy and adverse medicine interactions for older adults with multiple chronic conditions. ${ }^{10}$

In 2012, The American Board of Internal Medicine (ABIM) launched the Choosing Wisely (CW) initiative to identify unnecessary tests, treatments, and procedures. ${ }^{11}$ The American Geriatrics Society (AGS) released ten guidelines in 2013 (revised in 
2015), the third of which promotes less aggressive glycemic targets and reduction in pharmacologic therapy for older adults with type II diabetes. ${ }^{12-14}$ Despite the release of these guidelines, many providers are unaware of them, and several older adults with diabetes have a glycemic index that may be too tightly controlled. Furthermore, the CW campaign offers no tools to help achieve the recommended glycemic target ranges.

This study was developed to promote appropriate diabetes management in older adults using a unique approach: combining the power of behavioral economics (BE) with electronic health record (EHR) technology. BE combines insights from economics and psychology to recognize that humans are not perfectly rational agents who quickly synthesize all available evidence to make optimal choices (economics), but rather make predictable decision errors with known mechanisms (psychology). ${ }^{15-24}$ Historically, changing provider behavior has proven challenging, but novel $\mathrm{BE}$ tools suggest new mechanisms to influence clinicians. Interventions based on these ideas (termed "nudges") are increasingly applied in healthcare settings. ${ }^{25-31}$

Meanwhile, EHRs dominate the daily experience of clinicians, guiding documentation, ordering, data review, communication, and virtually all aspects of clinical care. Studies have used the EHR as a new channel for delivering reason-based interventions to influence clinician behavior. EHR tools that alert, suggest, and redirect clinical behavior, collectively called clinical decision support (CDS), have shown promise for influencing clinicians. ${ }^{32-36}$

The following details both the development and results of a pilot study implementing a behavioral economic electronic health record (BE-EHR) module to promote $\mathrm{CW}$ glycemic targets. We investigate how nudges embedded within EHRs may influence clinicians to improve the management of diabetes in older adults.

\section{METHODS}

This study was approved by the NYU School of Medicine Institutional Review Board (i17-01308).

In total, six CDS tools grounded in BE theory were developed for integration into the New York University Langone Health (NYULH) Epic EHR system. The tools, or "nudges," are visual or other cues to signal to clinicians that they are seeing a patient for whom CW guidelines are relevant. These "nudges" were piloted across five NYULH primary care and endocrinology clinics in the greater New York City area from June 12, 2018, through October 31, 2019. Practices were carefully selected for characteristics including the number of full-time providers and support staff, primary care focus, diverse patient sociodemographics and relevant older patient population with diabetes, location, and acceptance of insurance plans. Two of the five sites were selected as Vanguard practices due to willingness of practice leadership to serve as a test site for implementation of module prototypes and provide periodic feedback. The remaining three practices were designated as Pilot sites where BE-EHR components were implemented after feedback and refinement from initial implementation in the Vanguard sites.

\section{Study Population}

Eligible patients were those age 76 and older with type II diabetes (defined on the patient's "problem list" or as an "encounter diagnosis" within Epic). Patients who were not taking medication to treat diabetes, were allergic to metformin, or whose estimated glomerular filtration rate (eGFR) was less than 30 were excluded. To tailor the intervention appropriately, we developed an algorithm to categorize patients into one of three LE categories. This algorithm incorporated the patient's gender and age and used a weighted scoring approach for the number of comorbidities, ${ }^{37,38}$ as well as LE tables assuming chronic conditions using Medicare beneficiary data ${ }^{39}$ (see ESM). As shown in Table 1, the LE algorithm was built into the BE-EHR module to drive content of firings based on three target glycemic index ranges per the $\mathrm{CW}$ guideline. $^{12}$

Patients were then categorized as either $\mathrm{CW}$ compliant or noncompliant depending on their actual $\mathrm{HbAlc}$ relative to the target range in each LE category. Per the $\mathrm{CW}$ guideline, metformin was suggested for any patient currently taking a nonmetformin medication. However, CW noncompliance was only present if a patient's $\mathrm{HbAlc}$ was below the lower limit of the glycemic target threshold for their respective LE categorization.

Table 1 lists the definitions of CW compliance and noncompliance for each LE category. An alternative definition for $\mathrm{CW}$ compliance that includes undertreated patients in both the numerator and denominator, as well as corresponding results, can be found in the ESM.

\section{Nudge Development}

This study employed a pragmatic, user-centered approach to develop a BE-EHR module consisting of six individual components or "nudges" for implementation into Epic as a CDS tool. ${ }^{40}$ Based on quantitative and qualitative data collected, each nudge was developed iteratively, resulting in final versions with sufficient provider utilization and satisfaction.

As shown in Figure 1, the following steps aided in the development of each nudge for the BE-EHR module:

1. Semistructured interviews with key informants $(n=10$; expertise from physicians and medical directors working in clinical informatics or with the Epic system)

2. Two 2-h design thinking workshops (Spring 2018) with multidisciplinary groups of clinicians, informaticists, EHR analysts, product designers, and others to derive and refine initial module ideas

3. Site visits to the two Vanguard clinics, consisting of semistructured group interviews with clinic leaders and clinicians to elicit feedback on three draft module components 
Table 1 LE Categorizations and Corresponding CW Compliance and Noncompliance Definitions

\begin{tabular}{|c|c|}
\hline$\frac{\text { High Life Expectanc }}{\text { comorbidities and LE o }}$ & $\begin{array}{l}\text { healthy older adults with a limited number of } \\
0+\text { years; HbA1c target range of } 7-7.5 \%\end{array}$ \\
\hline CW Compliance: & $\begin{array}{l}\text { ratio of eligible patients with } \mathrm{HbA1c} \text { range: } \\
\frac{7.0-7.5 \%}{\leq 7.5 \%}\end{array}$ \\
\hline $\begin{array}{l}\text { CW Non- } \\
\text { compliance: }\end{array}$ & ratio of eligible patients with $\mathrm{HbA} 1 \mathrm{c}$ range: $\frac{<7.0 \%}{\leq 7.5 \%}$ \\
\hline$\frac{\text { Medium Life Expect }}{\text { comorbidities and LE o }}$ & $\begin{array}{l}\text { ancy: older adults with a moderate number of } \\
3+\text { to } 10 \text { years; } \mathrm{HbA} 1 \mathrm{c} \text { target range of } 7.5-8 \%\end{array}$ \\
\hline CW Compliance: & $\begin{array}{l}\text { ratio of eligible patients with } \mathrm{HbA1c} \text { range: } \\
\frac{7.5-8.0 \%}{\leq 8.0 \%}\end{array}$ \\
\hline $\begin{array}{l}\text { CW Non- } \\
\text { compliance: }\end{array}$ & ratio of eligible patients with $\mathrm{HbAlc}$ range: $\frac{<7.5 \%}{\leq 8.0 \%}$ \\
\hline $\begin{array}{l}\text { Low Life Expectancy: } \\
\text { years or less; HbAlc tai }\end{array}$ & $\begin{array}{l}\text { older adults with multiple comorbidities and LE of } 3 \\
\text { get range of } 8-9 \%\end{array}$ \\
\hline CW Compliance: & $\begin{array}{l}\text { ratio of eligible patients with HbA1c range: } \\
\frac{8.0-9.0 \%}{\leq 9.0 \%}\end{array}$ \\
\hline $\begin{array}{l}\text { CW } \\
\text { compliance: }\end{array}$ & ratio of eligible patients with $\mathrm{HbA} 1 \mathrm{c}$ range: $\frac{<8.0 \%}{\leq 9.0 \%}$ \\
\hline
\end{tabular}

Insights generated from these activities were recorded by research staff and summarized by usability theme and module component for rapid iteration of the prototype. Clinician considerations and motivation when treating patients in the target population, including compatibility with provider workflow, were also used to develop content ideas and message refinement for nudges.

After findings were incorporated into the prototype, the module was deployed in the two Vanguard sites. Utilizing Epic to identify encounters in which the module was likely to show, research staff arranged to observe these encounters to assess clinician use and interactions with the module in the live clinical settings on August 22, 27, and 29, 2018.

In September/October 2018, visits were made to the remaining three Pilot sites to conduct semistructured group interviews with clinic leaders and clinicians to elicit feedback on draft module component ideas. On October 24, 2018, four of the finalized BE-EHR module components were deployed in the two Vanguard and three Pilot clinics, with the remaining two nudges disseminated in December 2018 and April 2019. The final six BE-EHR module components are summarized in
Table 2 and described in detail below, including launch dates, triggers for activation, and BE principles utilized. Images of these nudges can be found in the ESM.

Nudge \#1-Tailored Advisory. This activates noninterruptively in Epic for any CW-noncompliant patient; it was launched on June 12, 2018, in Vanguard practices and on October 24, 2018, in Pilot sites. For each patient seen, clinicians can respond by clicking the "Agree with recommendation. Action taken" button, or by selecting the "Clinically inappropriate. Please explain" option, with space for free text comments. A response is not required. Clinicians had the option to suppress future nudge activations for a particular patient for half a year (182.5 days) with either of these acknowledgments.

Nudge \#2-Refill Protocol. This activates noninterruptively in Epic any time a refill for diabetes medication is generated for a patient 76+; it suggests that providers order metformin as an alternative for patients who are not already on this medication and to consider refilling at a lower dose or not at all for patients 


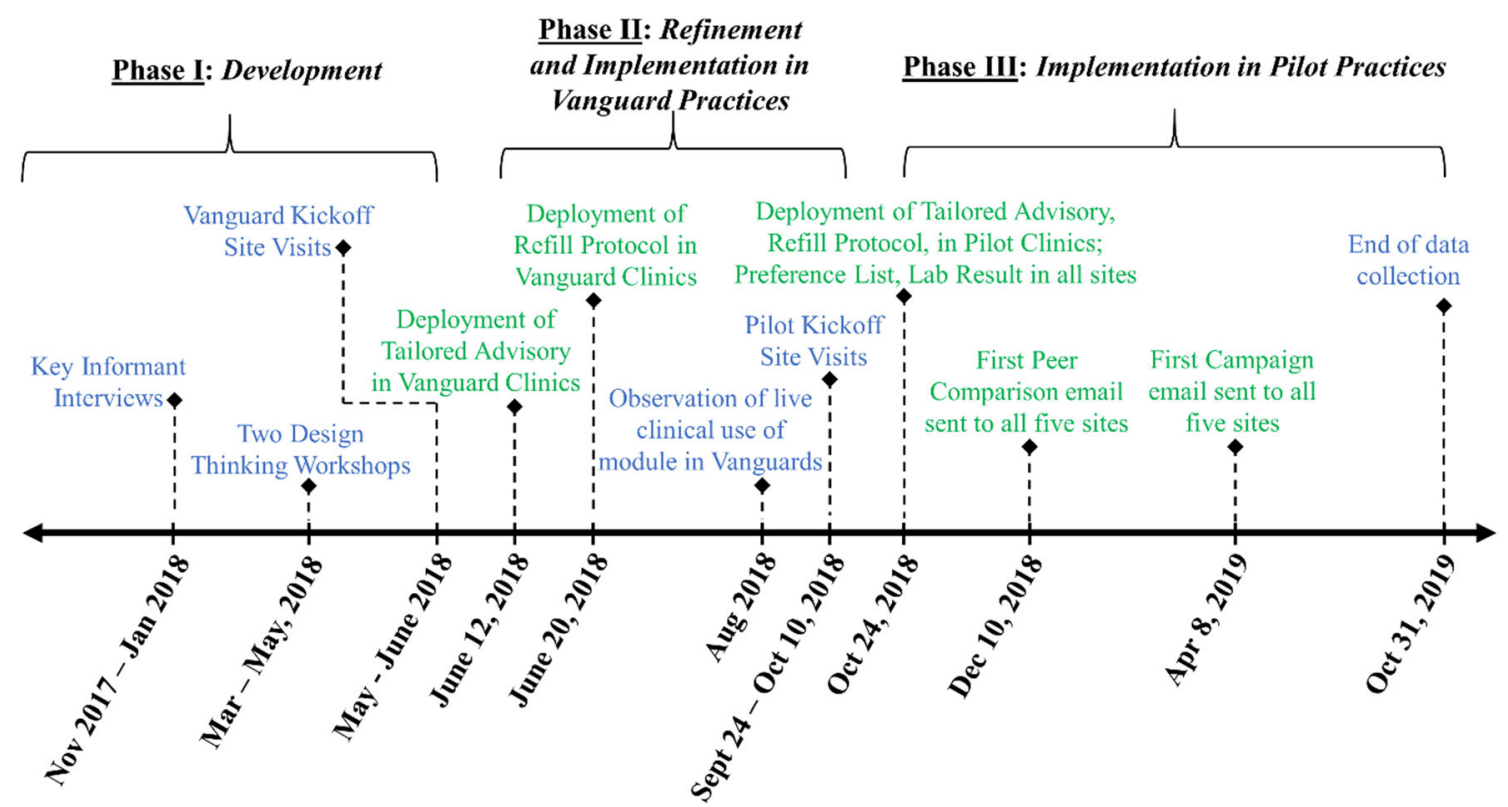

Figure 1 Timeline of BE-EHR module component development and implementation in Vanguard and Pilot practices. Blue text indicates development/refinement tasks and green text indicates implementation steps.

already taking metformin; this nudge was launched on June 20, 2018, in Vanguard sites and on October 24, 2018, in Pilot sites. The Refill Protocol also permitted providers to leave comments.

Nudge \#3-Preference List. This was built as a system-level nudge. Metformin is listed at the top of the page as the choice for "First-line Type 2 Diabetes," without restricting orders for non-metformin medications. This nudge was launched in both Vanguard and Pilot sites on October 24, 2018.

Nudge \#4-Lab Result. This activates noninterruptively in Epic whenever there is a new HbAlc lab result for a non-CWcompliant patient; the alert remains active in Epic for 7 days following the result. This nudge was launched in both Vanguard and Pilot sites on October 24, 2018.

For the four nudges listed above, a system modification was implemented on November 28, 2018, to fix a technical issue of the nudges activating multiple times for a single patient visit.
Nudge \#5-Peer Comparison. This was sent via a secured Microsoft Outlook account once per month beginning on December 10, 2018, and subsequently in 2019 on January 1, February 11, March 11, April 15, May 9, June 24, July 15, August 12, September 24, and October 28. The subject line of the email was "Message from the desk of Dr. [Insert Practice Director Name]" and the email content included three graphics: a $\mathrm{CW}$ compliance rate for the individual provider, a CW compliance rate for the clinician's practice site, and a CW compliance rate across all NYULH practices. Depending on whether the clinician's CW compliance rate was above or below the rate of their respective practice, the provider would receive either a "negative" or "positive" version of the accompanying text.

Nudge \#6-Campaign. Two separate workshops were used to design an educational email campaign bringing awareness of the CW guidelines to providers. The workshops included clinicians, researchers, and health services experts. Gameshow-themed

Table 2 BE-EHR Module Components

\begin{tabular}{|c|c|c|}
\hline Nudge & Description & BE principles* \\
\hline 1. Tailored advisory & $\begin{array}{l}\text { Alert window describing appropriate } \\
\text { treatment guidelines for older adults }\end{array}$ & $\begin{array}{l}\text { Framing, social norming, suggesting } \\
\text { alternatives, affirmation, emotional } \\
\text { appeal, accountable justification }\end{array}$ \\
\hline 2. Refill protocol & $\begin{array}{l}\text { Alert window appearing in refills section } \\
\text { describing appropriate treatment } \\
\text { guidelines for older adults }\end{array}$ & $\begin{array}{l}\text { Framing, social norming, suggesting } \\
\text { alternatives, affirmation, emotional appeal, } \\
\text { accountable justification }\end{array}$ \\
\hline 3. Preference list & Automatic defaulting of medication list & Defaults \\
\hline 4. Lab result & $\begin{array}{l}\text { Alert window appearing in lab results } \\
\text { section describing appropriate treatment } \\
\text { guidelines for older adults }\end{array}$ & $\begin{array}{l}\text { Framing, social norming, suggesting } \\
\text { alternatives, emotional appeal }\end{array}$ \\
\hline 5. Peer comparison & $\begin{array}{l}\text { Email with comparison of individual, } \\
\text { group, and system-wide performance }\end{array}$ & Social norming, competition \\
\hline 6. Campaign & Animated email with information on $\mathrm{CW}$ guidelines & Gamification, competition \\
\hline
\end{tabular}

*For definitions of these BE principles, please see ESM 
prototypes emerged from the design workshops. Prototype user testing was conducted by interviewing a total of 20 individuals including clinicians (internists, psychiatrists, cardiologists, primary care physicians) and researchers, across five different academic institutions in the greater New York City and Boston areas. The final Campaign toolkit for dissemination included three gameshow-themed animations inspired by The Price is Right $^{\mathrm{TM}}$, Jeopardy ${ }^{\mathrm{TM}}$, and Who Wants to Be a Millionaire ${ }^{\mathrm{TM}}$, as well as a flashcard deck that quizzes physicians on $\mathrm{CW}$ best practices. For each of the four animations, there were multiple versions of each that varied according to the three LE categories and information provided to bring awareness to the $\mathrm{CW}$ initiative. Clinicians were randomly assigned to receive one version of each animation per monthly Campaign email. The Price is

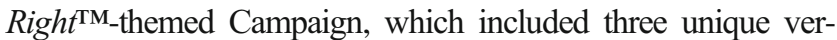
sions, was emailed to providers at all five practice sites on April 8 and August 15, 2019; the Jeopardy ${ }^{\mathrm{TM}}$-themed Campaign, which included four unique versions, was disseminated on May 6 and September 26, 2019; the Who Wants to Be a Millionaire ${ }^{\mathrm{TM}}$-themed Campaign, which included three unique versions, was sent on June 27 and October 31, 2019; finally, the flashcard deck, which included three unique versions, was emailed on July 18, 2019.

\section{Statistical Analysis}

The primary outcome was $\mathrm{CW}$ compliance, measured in consecutive 4-week intervals from February 14, 2018, through October 22, 2019. To compare CW compliance rates pre- and post-intervention, mean and $95 \%$ confidence intervals were computed for the four 4-week intervals just prior to launch in the Vanguard sites and compared to the most recent four 4week intervals of data collection. All analyses were performed using $\mathrm{R}$ version 3.4.3.

Utilization rates were collected for the Tailored Advisory, Refill Protocol, Preference List, and Lab Result nudges using an EHR-based report that tracked frequency of BE-EHR module component firings or placing of an order and action taken by the provider. These metrics were calculated per the number of unique providers and patients for each module, and when appropriate, stratified by gender, age, practice location, LE, and whether the patient received a metformin or non-

Table 3 Nudge Activation

\begin{tabular}{|c|c|c|c|c|c|c|}
\hline & & \multicolumn{5}{|l|}{ Nudge } \\
\hline & & $\begin{array}{l}\text { Tailored } \\
\text { Advisory }\end{array}$ & $\begin{array}{l}\text { Refill } \\
\text { Protocol }\end{array}$ & Lab Result & $\begin{array}{l}\text { Preference } \\
\text { List } \\
\text { (metformin) }\end{array}$ & $\begin{array}{l}\text { Preference List } \\
\text { (non- } \\
\text { metformin) }\end{array}$ \\
\hline Activations & $\begin{array}{l}\text { Total } \\
\text { Unique providers } \\
\text { Unique patients }\end{array}$ & $\begin{array}{l}3192 \\
60 \\
1033\end{array}$ & $\begin{array}{l}2332 \\
70 \\
796\end{array}$ & $\begin{array}{l}3445 \\
73 \\
813\end{array}$ & $\begin{array}{l}213 \\
43 \\
189\end{array}$ & $\begin{array}{l}360 \\
47 \\
272\end{array}$ \\
\hline \multicolumn{7}{|c|}{ Breakdown by number of unique patients } \\
\hline \multirow[t]{3}{*}{ Location } & $\begin{array}{l}\text { Vanguard } 1 \\
\text { Vanguard } 2\end{array}$ & $\begin{array}{l}121(11.7 \%) \\
220(21.3 \%)\end{array}$ & $\begin{array}{l}89(11.2 \%) \\
164(20.6 \%)\end{array}$ & $\begin{array}{l}92(11.3 \%) \\
167 \\
(20.5 \%)\end{array}$ & \multirow{14}{*}{\multicolumn{2}{|c|}{$\begin{array}{l}\text { Patient-level data was } \\
\text { not collected for the } \\
\text { Preference List nudge. }\end{array}$}} \\
\hline & $\begin{array}{l}\text { Pilot } 1 \\
\text { Pilot } 2\end{array}$ & $\begin{array}{l}131(12.7 \%) \\
220(21.3 \%)\end{array}$ & $\begin{array}{l}116(14.6 \%) \\
192(24.1 \%)\end{array}$ & $\begin{array}{l}95(11.7 \%) \\
195 \\
(24.0 \%)\end{array}$ & & \\
\hline & Pilot 3 & $341(33.0 \%)$ & $235(29.5 \%)$ & $\begin{array}{l}264 \\
(32.5 \%)\end{array}$ & & \\
\hline Gender & Female & $604(58.5 \%)$ & $466(58.5 \%)$ & $\begin{array}{l}475 \\
(58.4 \%)\end{array}$ & & \\
\hline \multirow[t]{4}{*}{ Age range } & 76-79 years & $332(32.1 \%)$ & $265(33.3 \%)$ & $\begin{array}{l}268 \\
(33.0 \%)\end{array}$ & & \\
\hline & $80-89$ years & $606(58.7 \%)$ & $461(57.9 \%)$ & $\begin{array}{l}483 \\
(59.4 \%)\end{array}$ & & \\
\hline & 90-99 years & $93(9.0 \%)$ & $69(8.7 \%)$ & $62(7.6 \%)$ & & \\
\hline & $100+$ years & $2(0.2 \%)$ & $1(0.1 \%)$ & $0(0 \%)$ & & \\
\hline \multirow[t]{3}{*}{ LE } & High & $676(65.4 \%)$ & $541(68.0 \%)$ & $\begin{array}{l}528 \\
(64.9 \%)\end{array}$ & & \\
\hline & Medium & $343(33.2 \%)$ & $245(30.8 \%)$ & $\begin{array}{l}271 \\
(33.3 \%)\end{array}$ & & \\
\hline & Low & $14(1.4 \%)$ & $10(1.3 \%)$ & $14(1.7 \%)$ & & \\
\hline \multirow[t]{2}{*}{ Medication status } & Metformin alert & $534(51.7 \%)$ & $359(45.1 \%)$ & $\begin{array}{l}475 \\
(58.4 \%)\end{array}$ & & \\
\hline & $\begin{array}{l}\text { Non-metformin } \\
\text { alert }\end{array}$ & $499(48.3 \%)$ & $437(54.9 \%)$ & $\begin{array}{l}338 \\
(41.6 \%)\end{array}$ & & \\
\hline $\begin{array}{l}\text { Provider } \\
\text { feedback }\end{array}$ & Comments & $\begin{array}{l}126 \text { total (109 unique } \\
\text { patients) }\end{array}$ & 0 & $\mathrm{~N} / \mathrm{A}$ & & \\
\hline
\end{tabular}

(Top half) Frequency of activation of nudges \#1 through \#4 from June 12, 2018 (date of earliest nudge activation), through October 17, 2019. Counts are provided in total and by the number of unique providers and patients who received each alert. The Preference List is further stratified by orders placed for metformin or non-metformin medications within Epic. (Bottom half) Stratification of the number of unique patients who received nudges \#1 through \#3 by practice location, gender, age range, LE categorization, whether the patient received a metformin or non-metformin-based medication alert, and number of comments left by providers 


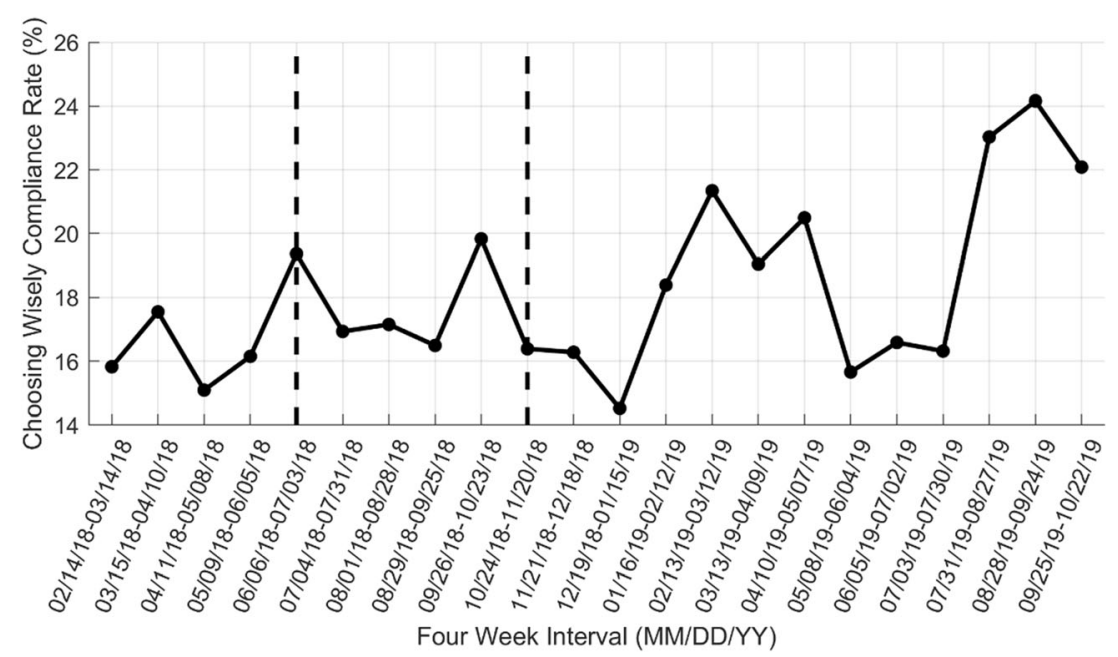

Figure $2 \mathrm{CW}$ compliance rates in 4-week discrete intervals across all five practice locations. Data to the left of the first vertical dashed bar indicate baseline CW compliance rates prior to initial launch in only the Vanguard practices. Data to the right of the second vertical bar indicate $\mathrm{CW}$ compliance rates after activation of the nudges at all five practices.

metformin alert. Because the Peer Comparison and Campaign nudges were disseminated directly by the research team on a monthly schedule, rather than activated by patient visits within Epic, utilization rates for these nudges were reported according to the scheduled dissemination frequencies.

Although individual activation rates for select BE-EHR components were tracked, the goal was not to discern which nudges were individually more effective at influencing clinicians to adopt the $\mathrm{CW}$ guideline, but instead to assess whether the toolbox comprising the collection of nudges incorporated throughout NYULH Epic EHR and delivered electronically via email could increase $\mathrm{CW}$ compliance among patients.

\section{RESULTS}

\section{Utilization of Individual BE-EHR Module Components}

Table 3 summarizes the number of times nudges \#1-4 (see Table 2) were activated. Among the Epic-based alerts, the Tailored Advisory activated for the most unique patients
(1033), with the majority in the high LE category (65.4\%). Roughly half of the alerts were for patients already taking metformin $(51.7 \%)$, and providers left comments for 109 $(10.6 \%)$ of the patients. The Lab Result activated the greatest number of times (3445), but for fewer unique patients (813). Patient-level activation rates were not collected for the Peer Comparison or Campaign nudges because these two BE-EHR module components were disseminated monthly to clinicians rather than triggered within Epic during patient visits. However, provider-level dissemination frequencies were collected for the Campaign nudge and are detailed in the ESM.

\section{Overall CW Compliance}

Figure 2 shows $\mathrm{CW}$ compliance rates across all five sites in discrete 4-week intervals between February 14, 2018, and October 22, 2019, and Table 4 provides the corresponding mean and $95 \%$ confidence intervals pre- and postintervention.

Because the confidence interval for the 16 -week $\mathrm{CW}$ compliance rate at baseline just slightly overlaps with the confidence interval for the 16 -week $\mathrm{CW}$ compliance rate

Table 4 CW Compliance

\begin{tabular}{llll}
\hline \hline & Dates & $\begin{array}{l}\text { Mean } \\
\text { (95\% CI) }\end{array}$ & $\begin{array}{l}\text { Number of } \\
\text { patients }\end{array}$ \\
\hline Baseline & February 14-March 14, 2018 & $15.8 \%(11.8 \%, 19.9 \%)$ & 316 \\
& March 15-April 10, 2018 & $17.5 \%(13.3 \%, 21.8 \%)$ & 302 \\
& April 11-May 8, 2018 & $15.1 \%(11.3 \%, 18.9 \%)$ & 338 \\
& May 9-June 5, 2018 & $16.1 \%(12.1 \%, 20.2 \%)$ & 322 \\
Most recent & February 14-June 5, 2018 & $16.1 \%(14.1 \%, 18.1 \%)$ & 1278 \\
& July 3-July 30, 2019 & $16.3 \%(11.1 \%, 21.6 \%)$ & 178 \\
& July 31-August 27, 2019 & $23.0 \%(16.9 \%, 29.2 \%)$ & 149 \\
& August 28-September 24, 2019 & $24.2 \%(17.3 \%, 31.0 \%)$ & 63 \\
& September 25-October 22, 2019 & $22.1 \%(15.7 \%, 28.5 \%)$ & 680 \\
\hline
\end{tabular}

(Top) CW compliance, mean (95\% CI), at baseline for each of the first four 4-week intervals prior to nudge launch in the Vanguard sites and overall baseline CW compliance rate across all 16 weeks. (Bottom) CW compliance, mean (95\% CI), in the most recent four 4-week intervals after nudge deployment at all five practices, as well as the overall postintervention $C W$ compliance rate across all 16 weeks 
postintervention (Table 4), we cautiously interpret this result as an indication of improvement in $\mathrm{CW}$ compliance rather than claiming statistical significance. We anticipate that $\mathrm{CW}$ compliance will continue to increase over time as more patients are seen and return to the clinic for follow-up visits. A clinical change in $\mathrm{HbAlc}$ lab result is expected to occur no sooner than 3 months after the last lab test, ${ }^{41}$ and only 1092 (71.1\%) of the 1536 patients with a nonmissing $\mathrm{CW}$ status that were seen in the five practices between October 24, 2018, and October 31, 2019 had a new HbAlc lab result 90 days or more since their first lab test.

\section{DISCUSSION}

Integrating BE and EHRs using CDS tools is a novel approach to improving adherence to guidelines that also seeks to minimize negative impacts on clinical workflow and cognitive load. Recent studies have utilized similar approaches including integrating three $\mathrm{BE}$ concepts (suggested alternatives, accountable justification, and peer comparisons) into the EHR to effectively reduce the rate of inappropriate antibiotic prescriptions for acute respiratory infections, ${ }^{27}$ using active choice within the EHR to significantly increase influenza vaccination rates, ${ }^{42}$ physician ordering, and patient completion of high-value cancer screening tests, ${ }^{43}$ and nudging primary care physicians to increase guideline-concordant statin prescribing. ${ }^{44}$ The present work adds to the literature by being the first to leverage $\mathrm{BE}$ and EHRs for $\mathrm{CW}$-driven deprescribing, specifically in older adults with diabetes. Furthermore, this is one of the first studies to develop a multicomponent intervention toolbox comprising several nudges, and shows promise for managing a chronic disease rather than a one-time clinical event.

Results showed a $5.1 \%$ increase in CW compliance from a 16week period prior to the first nudge launch in the Vanguard clinics to the most recent 16 weeks of data collection, a noteworthy increase considering patient- and clinician-level barriers surrounding deprescribing medications (e.g., reluctance of patients to stop medications once viewed as necessary, or limited incentives for clinicians to stop medications). ${ }^{45}$ Furthermore, the BEEHR module shows promise considering that four of the six BEEHR module components were not active across all five clinics until October 24, 2018, and that through October 31, 2019, only $\sim 71 \%$ of the patient population had a return visit and new $\mathrm{HbA} 1 \mathrm{c}$ lab test at least 90 days later. ${ }^{41}$

We acknowledge the limitations of the current research. The present work tested the effectiveness of the BE-EHR module as a whole, rather than individual components, suggesting that the combination of BE and EHR CDS tools offers promise for improving diabetes management in older adults. Future research is necessary to determine which specific behavioral economics principles may be most effective at limiting overtreatment in elderly adults with diabetes.
As a pilot study, the five clinics were not randomly selected and there was no control group. To rigorously assess the effectiveness of the BE-EHR module, a full RCT will commence in winter 2020, including randomization of 66 NYULH practices. The current short study duration was unable to detect physician habituation over time. Thus, the RCT will run for 18 months to assess the long-term effects of the intervention.

Although we collected utilization rates, we currently have limited evidence (other than if a physician were to leave a comment) about the degree to which clinicians are viewing or interacting with the BE-EHR module components. In the $\mathrm{RCT}$, we plan to monitor provider interaction by collecting data on where clinicians click within Epic and from email read receipts.

Furthermore, utilization rates indicated that almost twothirds of all patients were in the high LE category, with less than $2 \%$ in the low LE group, suggesting a potential bias toward healthy older adults more frequently visiting clinics.

We acknowledge that the $\mathrm{CW}$ campaign includes society recommendations, and the "reasonable targets" are guidelines rather than explicit treatment ranges. As with any medical care, we support clinicians utilizing all available information to provide optimal treatment to patients.

Finally, since the release of the AGS CW guidelines, multiple trials have demonstrated diabetes and cardiovascular benefits of SGLT-2i and GLP-1 with minimal risk for hypoglycemic-related morbidity. ${ }^{46}$ As this evidence amasses, we anticipate that guidelines will change, and we will update the tools as appropriate. Regardless, we believe the tools tested in this intervention will be applicable to many other use cases and are adaptable to changing guidelines.

Acknowledgments: We thank Catherine Dinh-Le, Katherine Driscoll, and Steven Robinson for their work in the development of the Campaign BE-EHR module component.

Corresponding Author: Hayley M. Belli, PhD; Division of Biostatistics, Department of Population Health, New York University School of Medicine, New York, NY, USA (e-mail: Hayley.Belli@nyulangone.org).

Funding Information This research was supported by NIH NIA Award R21 AG057382 awarded to ABT and DMM. HMB was supported in part by NIH 5TL1TROO1447.

\section{Compliance with Ethical Standards:}

Conflict of Interest: The authors declare that they do not have a conflict of interest.

\section{REFERENCES}

1. Gerstein HC, Miller ME, Byington RP, et al. Effects of intensive glucose lowering in type 2 diabetes. N Engl J Med 2008;358(24):2545-2559.

2. Patel A, MacMahon S, Chalmers J, et al. Intensive blood glucose control and vascular outcomes in patients with type 2 diabetes. N Engl J Med 2008;358(24):2560-2572. 
3. Duckworth W, Abraira C, Moritz T, et al. Glucose control and vascular complications in veterans with type 2 diabetes. $\mathrm{N}$ Engl $\mathrm{J}$ Med 2009;360(2): 129-139.

4. Workgroup AGSCW. American Geriatrics Society identifies five things that healthcare providers and patients should question. J Am Geriatr Soc 2013;61(4):622-631.

5. Shorr RI, Ray WA, Daugherty JR, Griffin MR. Incidence and risk factors for serious hypoglycemia in older persons using insulin or sulfonylureas. Arch Intern Med 1997;157(15):1681-1686.

6. Bremer JP, Jauch-Chara K, Hallschmid M, Schmid S, Schultes B. Hypoglycemia unawareness in older compared with middle-aged patients with type 2 diabetes. Diabetes Care 2009;32(8): 1513-1517.

7. Huang ES, Laiteerapong N, Liu JY, John PM, Moffet HH, Karter AJ. Rates of complications and mortality in older patients with diabetes mellitus: the diabetes and aging study. JAMA Intern Med 2014;174(2):251-258

8. American Diabetes A 11. Older Adults Diabetes Care 2017;40(Suppl 1):S99-S104

9. Geller AI, Shehab N, Lovegrove $\mathbf{M C}$, et al. National estimates of insulinrelated hypoglycemia and errors leading to emergency department visits and hospitalizations. JAMA Intern Med 2014; 174(5):678-686.

10. Boyd CM, Darer J, Boult C, Fried LP, Boult L, Wu AW. Clinical practice guidelines and quality of care for older patients with multiple comorbid diseases: implications for pay for performance. JAMA. 2005;294(6):716724 .

11. ABIM. Choosing Wisely: promoting conversations between patients and clinicians. 2019; https://www.choosingwisely.org/. Accessed July 20 , 2019.

12. AGS. Choosing Wisely: an initiative of the ABIM Foundation. 2017; http://www.choosingwisely.org/clinician-lists/american-geriatrics-society-medication-to-control-type-2-diabetes/. Accessed July 20, 2019.

13. American Geriatrics Society Expert Panel on Care of Older Adults with Diabetes M, Moreno G, Mangione CM, Kimbro L, Vaisberg E. Guidelines abstracted from the american geriatrics society guidelines for improving the care of older adults with diabetes mellitus: 2013 update J Am Geriatr Soc 2013;61(11):2020-2026

14. Workgroup AGSCW. American Geriatrics Society identifies another five things that healthcare providers and patients should question. J Am Geriatr Soc 2014;62(5):950-960.

15. Ariely D. Predictably Irrational. New York: HarperCollins; 2008.

16. Camerer C, Issacharoff S, Loewenstein G, O'Donoghue T, Rabin M. Regulation for conservatives: behavioral economics and the case for “asymmetric paternalism”. Univ Pa Law Rev 2003;101:1211-1254.

17. Connolly T, Butler D. Regret in economic and psychological theories of choice. J Behav Decis Mak 2006; 19(2):139-154.

18. Kahneman D, Tversky A. Judgment under uncertainty: heuristics and biases. Science. 1974; 185(4157):1124-1131.

19. Kahneman D, Tversky A. Prospect theory: an analysis of decision under risk. Econometrica. 1979;47(2):263-291.

20. Loewenstein G, John L, Volpp K. Using decision errors to help people help themselves. Chapter 21 in The Behavioral Foundations of Public Policy, edited by Eldar Shafir. Princeton University Press, 2012:361-79.

21. Mullainathan S, Thaler R. Behavioral Economics. International Encyclopedia of the Social \& Behavioral Sciences. Elsevier; 2001:1094-1100.

22. Thaler R, Sunstein C. Libertarian paternalism. Am Econ Rev 2003;93:175-179.

23. Thaler R, Tversky A, Kahneman D, Schwartz A. The effect of myopia and loss aversion on risk taking: an experimental test. $\mathrm{Q} \mathrm{J}$ Econ 1997; 112(2):647-661.

24. Tversky A, Kahneman D. Loss aversion in riskless choice: a referencedependent model. Q J Econ 1991;106(4):1029-1061.

25. Chapman GB, Coups EJ. Emotions and preventive health behavior: worry, regret, and influenza vaccination. Health Psychol 2006;25(1):82 90.

26. Marti J, Bachhuber M, Feingold J, Meads D, Richards M, Hennessy S Financial incentives to discontinue long-term benzodiazepine use: discrete choice experiment investigating patient preferences and willingness to participate. BMJ Open 2017;7(10):e016229.
27. Meeker D, Linder JA, Fox CR, et al. Effect of Behavioral interventions on inappropriate antibiotic prescribing among primary care practices: a randomized clinical trial. JAMA. 2016;315(6):562-570.

28. Thaler R, Sunstein C. Nudge: Improving Decisions about Health, Wealth, and Happiness. 2nd ed. New York: Penguin Books; 2009.

29. Loewenstein G, Brennan T, Volpp KG. Asymmetric paternalism to improve health behaviors. JAMA. 2007;298(20):2415-2417.

30. Volpp KG, Loewenstein G, Troxel AB, et al. A test of financial incentives to improve warfarin adherence. BMC Health Serv Res 2008;8(1):272.

31. Kimmel SE, Troxel AB, Loewenstein G, et al. Randomized trial of lottery-based incentives to improve warfarin adherence. Am Heart J 2012;164(2):268-274.

32. Osheroff JA, Teich JM, Middleton B, Steen EB, Wright A, Detmer DE A roadmap for national action on clinical decision support. J Am Med Inform Assoc 2007; 14(2):141-145.

33. Jaspers MW, Smeulers M, Vermeulen H, Peute LW. Effects of clinical decision-support systems on practitioner performance and patient outcomes: a synthesis of high-quality systematic review findings. J Am Med Inform Assoc 2011;18(3):327-334.

34. Bright TJ, Wong A, Dhurjati R, et al. Effect of clinical decision-support systems: a systematic review. Ann Intern Med 2012;157(1):29-43.

35. Moja L, Kwag KH, Lytras T, et al. Effectiveness of computerized decision support systems linked to electronic health records: a systematic review and meta-analysis. Am J Public Health 2014;104(12):e12-22.

36. Khalifa M, Zabani I. Improving utilization of clinical decision support systems by reducing alert fatigue: strategies and recommendations. Stud Health Technol Inform 2016;226:51-4.

37. Charlson ME, Pompei P, Ales KL, MacKenzie CR. A new method of classifying prognostic comorbidity in longitudinal studies: development and validation. J Chronic Dis 1987; 40(5): 373-383.

38. Guan H, Li B, Couris CM, Fushimi K, Graham P, Hider P, Januel JM Sundararajan V. Updating and validating the Charlson comorbidity index and score for risk adjustment in hospital discharge abstracts using data from 6 countries. Am J Epidemiol 2011; 173(6): 676-682.

39. DuGoff EH, Canudas-Romo V, Buttorff C, Leff B, Anderson GF Multiple chronic conditions and life expectancy: a life table analysis. Med Care 2014; 52(8): 688-694.

40. Chokshi S, Belli, HM, Troxel, AB, et al. Designing for implementation: user-centered development and pilot testing of a behavioral economicinspired electronic health record clinical decision support module. Pilot and Feasibility Studies 2019; 5:28.

41. Sacks DB, Bruns DE, Goldstein DE, Maclaren NK, McDonald JM, Parrott M. Guidelines and recommendations for laboratory analysis in the diagnosis and management of diabetes mellitus. Clin Chem 2002;48(3):436-472.

42. Patel MS, Volpp KG, Small DS, et al. Using active choice within the electronic health record to increase influenza vaccination rates. J Gen Intern Med 2017;32(7):790-795.

43. Patel MS, Volpp KG, Small DS, et al. Using active choice within the electronic health record to increase physician ordering and patient completion of high-value cancer screening tests. Healthc (Amst) 2016;4(4):340-345.

44. Patel MS, Kurtzman GW, Kannan S, et al. Effect of automated patient dashboard using active choice and peer comparison performance feedback to physicians on statin prescribing: the PRESCRIBE cluster randomized clinical trial. JAMA Netw Open 2018;1(3):e180818

45. Linsky A, Gellad WF, Linder JA, Friedberg MW. Advancing the science of deprescribing: a novel comprehensive conceptual framework. J Am Geriatr Soc 2019;67:2018-2022.

46. Hussein $\mathbf{H}$, Zaccardi $\mathbf{F}$, Khunti $\mathbf{K}$, et al. Efficacy and tolerability of sodium-glucose co-transporter-2 inhibitors and glucagon-like peptide-1 receptor agonists: a systematic review and network meta-analysis. Diabetes Obes Metab 2020; https://doi.org/10.1111/dom. 14008.

Publisher's Note Springer Nature remains neutral with regard to jurisdictional claims in published maps and institutional affiliations. 